\title{
Observing Galaxy Clusters with eROSITA: Simulations
}

\author{
J. Hölzl, J. Wilms, I. Kreykenbohm, Ch. Schmid, \\ Ch. Grossberger, M. Wille, W. Eikmann, T. Brand
}

\begin{abstract}
The eROSITA instrument on board the Russian Spectrum Roentgen Gamma spacecraft, which will be launched in 2013, will conduct an all sky survey in X-rays. A main objective of the survey is to observe galaxy clusters in order to constrain cosmological parameters and to obtain further knowledge about dark matter and dark energy. For the simulation of the eROSITA survey we present a Monte-Carlo code generating a mock catalogue of galaxy clusters distributed according to the mass function of [1]. The simulation generates the celestial coordinates as well as the cluster mass and redshift. From these parameters, the observed intensity and angular diameter are derived. These are used to scale Chandra cluster images as input for the survey-simulation.
\end{abstract}

Keywords: Galaxy clusters, cosmology, eROSITA, simulation.

\section{Introduction}

In recent years, our knowledge about the cosmological parameters has increased significantly through, e.g., precision measurements of the cosmic microwave background [2] and the Supernova Cosmology Project [3]. Measurements of the expansion history of our universe have provided evidence for the existence of a dark energy component which dominates all other contents of the universe and drives expansion [4]. A complementary method for measuring the cosmological parameters is to perform observations of large-scale structures. Galaxy clusters form in areas overdense with respect to the mean density of the universe and therefore trace the large-scale structure, such that a statistically complete sample of clusters provides us with information about the cosmological parameters [4]. By combining different measurements, degeneracies of parameters can be broken [4]. The mass function of clusters depends on the density parameter $\Omega_{\mathrm{m}}$ and the amplitude of the primordial power spectrum $\sigma_{8}$. The evolution of the mass function and also the amplitude and the shape of the power spectrum of the spatial cluster distribution $P(k)[5]$ are strongly influenced by dark matter and dark energy [4]. The baryonic acoustic oscillations, which enable the curvature of space to be measured, are also imprinted on the large-scale structure. In the following we present a simulation generating a catalogue of galaxy clusters, which is used as an input for the complete simulation of the eROSITA all sky survey [6].

Galaxy clusters are the largest virialized structures in the universe. The space between the galaxies is filled with an intracluster medium with temperatures of several tens of millions of degrees, which causes X-ray emission in the energy band of $\sim 2-15 \mathrm{keV}$. The gas traces the gravitational potential of the cluster, therefore the total mass of the cluster can be calculated by measuring the temperature and density profile [7].

\section{2 eROSITA}

eROSITA (extended ROentgen Survey with an Imaging Telescope Array) is one of two instruments on the Russian Spectrum Roentgen Gamma mission [8]. It consists of seven co-aligned identical Wolter-I X-ray telescopes with 54 nested mirrors each. Each of the Wolter telescopes is equipped with an identical pnCCD camera developed by the MPI Halbleiterlabor. The pnCCDs, which are backsideilluminated Charge Coupled Devices (CCDs), are advanced versions of the pnCCDs flying on XMMNewton [9]. The main science drivers of eROSITA are High Precision Cosmology, i.e., determination of the cosmological parameters independent of Cosmic Microwave Background and Supernova measurements, and the study of dark matter and dark energy. eROSITA will perform a deep all sky survey for four years, followed by pointed observations of selected fields. The flux limit will be at least one order of magnitude lower than the flux limit of the ROSAT All Sky Survey [10]. With an effective area of $\sim 1500 \mathrm{~cm}^{2}$ at $1.5 \mathrm{keV}$, eROSITA will detect about 50000-100000 galaxy clusters [4].

\section{Mass function}

As a cluster mass function, the function from [1] was used (Fig. 1):

$$
\frac{\mathrm{d} n}{\mathrm{~d} M}=f(\sigma) \frac{\bar{\rho}_{\mathrm{m}}}{M} \frac{\mathrm{d} \ln \sigma^{-1}}{\mathrm{~d} M}
$$




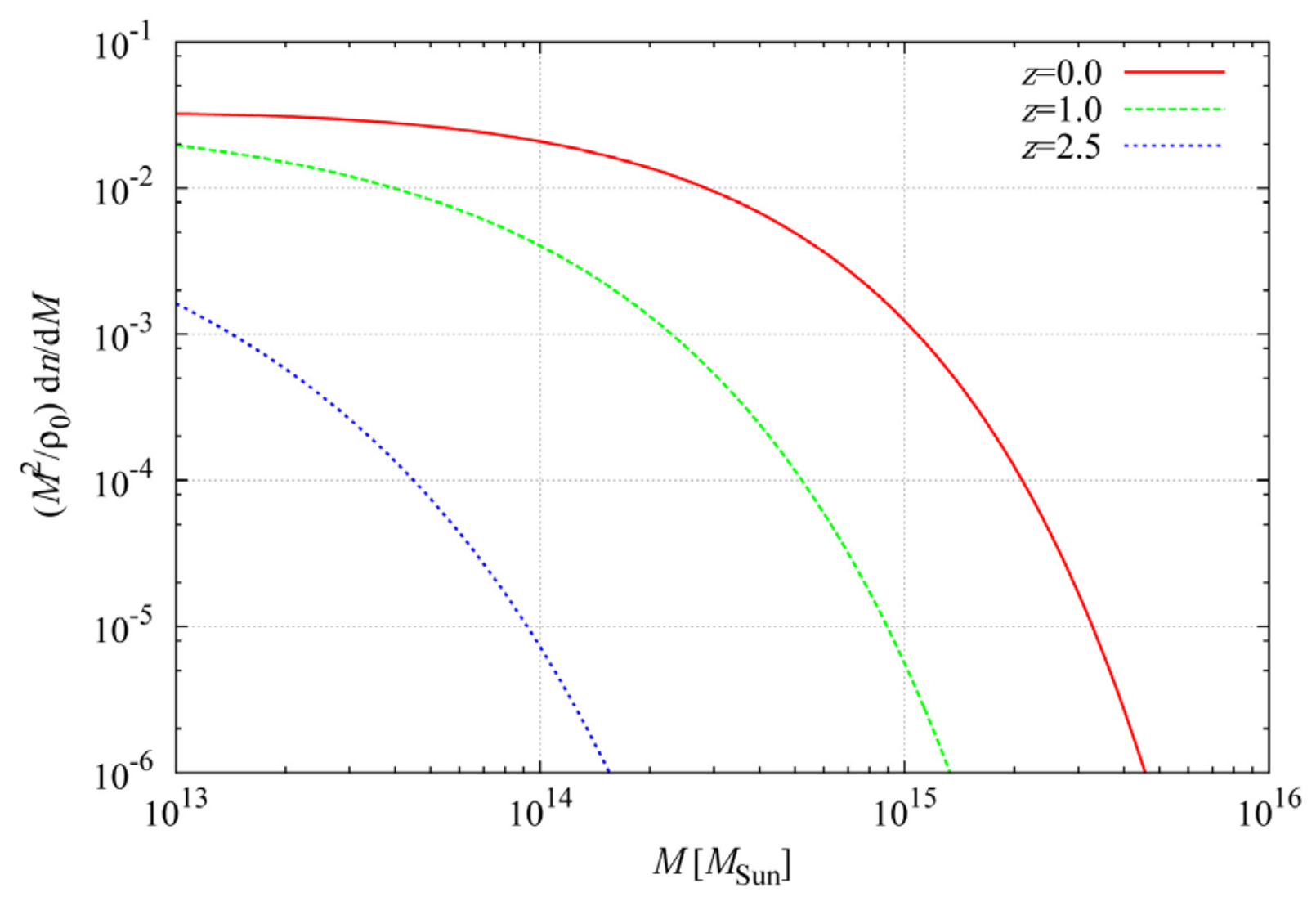

Fig. 1: Halo mass function from [1] for redshifts of 0,1 and 2.5 for halos with an overdensity of 500 relative to the critical density at redshift $z$. The $y$-axis is plotted with a factor $M^{2} / \rho_{0}$. The function shows a strong redshift evolution, the number density of clusters decreases with increasing redshift

where $n=\frac{\mathrm{d} N}{\mathrm{~d} V}$ is the number density of halos, $\bar{\rho}_{m}$ is the mean matter density of the universe, and $\sigma$ is the root mean square of the linear matter power spectrum at redshift $z$. The function $f(\sigma)$ has been calibrated by $N$-body simulations for redshifts up to $z=2.5$ [1]. Clusters are identified as isolated density peaks and the halo mass is calculated in a spherical area around the peak enclosing a specified overdensity. [1] describes the redshift evolution of the mass function through interpolation formulae or, alternatively, splines for the fitting parameters $f(\sigma)$. The redshift also enters through $\sigma(z)$.

\section{Simulation}

As a lower limit for the cluster mass we chose $10^{13} M_{\odot}$. Up to a redshift of $z=2.5$, the mass function gives about 23 million clusters with a mass larger than the limit. In the first step, the celestial coordinates, redshift, and mass were generated via the rejection sampling method [12]. The $L_{\mathrm{X}}-M$ relation by [11] was used to obtain the luminosity as a function of the mass. After sampling the catalogue, it was converted into a FITS file to be used as an input for the simulation of the survey, which is performed with the Simulation of X-ray Telescopes (SIXT) software [6]. Each entry of the catalogue links to an Chandra image of a galaxy cluster provided by Reiprich (private communication), which is scaled in size and luminosity according to the corresponding mass and redshift generated in the simulation, and to a spectrum.

\section{Summary and conclusions}

We generated a mock catalogue of galaxy clusters using a Monte-Carlo code. The final source catalogue contains Chandra-images of galaxy clusters, which are scaled in luminosity and diameter according to the mass and redshift sampled by the simulation. The next step is to take the correlationfunction, which is the Fourier-transform of the power spectrum [5], into account when sampling the cluster positions such that the celestial coordinates are not longer independent of redshift and mass (the mass enters because of the redshift evolution of the mass function). This will allow us to perform a realistic simulation of eROSITA's observing program. 
This research was funded by the Bundesministerium für Wirtschaft und Technologie under Deutsches Zentrum für Luft- und Raumfahrt grant numbers 50 QR 0903 and 50 OR 0801.

\section{References}

[1] Tinker, J., Kravtsov, A. V., Klypin, A., et al.: Astrophys. J. 688, 709, 2008.

[2] Komatsu, E., Smith, K. M., Dunkley, J., et al.: Astrophys. J. Suppl. Ser. 192, 18, 2011.

[3] Perlmutter, S., Schmidt, B. P.: In K. Weiler (ed.) Supernovae and Gamma-Ray Bursters. Lecture Notes in Physics, Berlin Springer Verlag, 598, 195, 2003.

[4] Predehl, P., Hasinger, G., Böhringer, H., et al.: In Space Telescopes and Instrumentation II: Ultraviolet to Gamma Ray. Proc. SPIE 6266, 62660P, 2006.

[5] Peacock, J. A.: Cosmological Physics. Cambridge : Cambridge University Press, 1999.

[6] Schmid, C., Martin, M., Wilms, J., et al.: X-ray Astronomy 2009, AIP Conf. Proc., 1248 , 591, 2010.
[7] Trümper, J. E., Hasinger, G. (eds.): The Universe in X-Rays (Astronomy and Astrophysics Library). Berlin : Springer, 2008.

[8] Pavlinsky, M., Sunyaev, R., Churazov, E., et al.: In Optics for EUV, X-Ray, and Gamma- Ray Astronomy IV. Proc. SPIE 7437, 743708, 2009.

[9] Strüder, L., Briel, U., Dennerl, K., et al.: Astron. Astrophys. 365, L18, 2001.

[10] Voges, W.: Adv. Space Res. 13, 391, 1993.

[11] Vikhlinin, A., Burenin, R. A., Ebeling, H., et al.: Astrophys. J. 692, 1 033, 2009.

[12] Deák, I.: Random Number Generators and Simulation. Budapest : Akademiai Kiado, 1990.

Johannes Hölzl

Jörn Wilms

Ingo Kreykenbohm

Christian Schmid

Christoph Grossberger

Michael Wille

Wiebke Eikmann

Thorsten Brand

Dr. Karl Remeis-Sternwarte/ECAP

Universität Erlangen-Nürnberg, Germany 